\title{
A Brief Overview of Techniques for Modulating Neuroendocrine and Other Neural Systems
}

\author{
Maryem Manzoor and Donald Pfaff
}

\begin{abstract}
The history of experimental approaches to the nervous system forms the backdrop for new opportunities of using stem cell technologies in neuroendocrine systems. The emphasis of this chapter is on attempts at therapeutic maneuvers.
\end{abstract}

\section{A Brief View of the Oldest, Most Primitive Approaches}

No one uncovers the historical roots, the origins of ancient neuroscience, better than Stanley Finger of the Washington University School of Medicine. Egyptians whose names have been lost, writing during the age of the Pyramids, treated "involved individuals who suffered from head injuries. The descriptions revealed that early Egyptian physicians were aware that symptoms of central nervous system injuries could occur far from the locus of the damage." The Greek physician Alcmaeon (around the fifth century BCE) did various dissections and "proposed that the brain was the central organ of sensation and thought." But things got serious when the Greek anatomist Galen (AD 130-200) numbered the cranial nerves, distinguished sensory and motor pathways, distinguished the cerebellum from the cortex, and described the autonomic ganglia that control our viscera.

The historical origins of the information on sensory pathways begin with studies of the visual system that "described two distinct types of endings (rods and cones) in the retina" and later, in fact, the discovery of one of the visual pigments, rhodopsin. Anatomical studies then proceeded to the visual pathways, both the direct "reflex action" pathways to the superior colliculus and to the classical thalamo-cortical system. In turn, one contrasts vision with olfaction, which does not use the thalamus to signal to the cortex. According to Finger, "until the second half of the eighteenth century, air was viewed as an element and passive carrier of foreign particles that could affect the health of an organism." Putrid smells were avoided. Soon the adequate stimulus for olfaction as particles in the air was

M. Manzoor • D. Pfaff ( $($ )

The Rockefeller University, New York, NY, USA

e-mail: pfaff@mail.rockefeller.edu 
recognized. It was known that olfactory receptors were in the nose, but the exact locations of the receptor-bearing cells were not known until the end of the nineteenth century. As with vision, investigations then proceeded to the central olfactory pathways in the brain.

Some of the initial findings reported paralysis on the side of the body that was opposite to brain damage that was limited to the cerebral cortex. Theorists supposed that the motor cortex was toward the front of the brain. But, in Finger's words, the "unequivocal experimental confirmation of a 'motor' cortex' electrically stimulated that part of cortex and caused movement." Confirming their results, subsequent removal of that part of the cortex of laboratory dogs led to motor deficits. Then, neurophysiologists would go on to define motor cortex precisely and to describe the motor tracts that lead from the forebrain toward the spinal cord.

Early ideas about emotion emphasized our visceral nervous systems, including both the sympathetic nervous system (raising blood pressure, heart rate, etc.) and the parasympathetic nervous system (usually the opposite effects of the sympathetic). In fact, the great psychologist/philosopher William James, at the end of the nineteenth century, actually proposed that we feel emotions consequent to changes in the autonomic nervous systems - feelings secondary to vascular changes. Walter Bradford Cannon and Philip Bard (at Johns Hopkins University) took a more straightforward view because they were able to stimulate the hypothalamus and directly cause emotional changes in experimental animals, changes like the induction of rage behavior. In subsequent years, the circuitry of the forebrain connected intimately to the hypothalamus (where we have done most of our work) proved to be essential for the performance of all emotional and motivated behaviors.

In the nineteenth century, clinicians had to deduce "how the brain works" by observing how behavioral capacities changed after brain damage. A special case was the British neurologist John Hughlings Jackson, who inferred which brain centers were "higher" and which "lower" by carefully noting how certain epileptic seizures in a given patient changed across time.

\section{The Disciplines}

Historically, the temporal order of accomplishment and understanding of brain function was that structure (morphology) led the way, followed by physiology (electrical recording), then chemistry (neurotransmitters), and now both genetics and computational neuroscience (in the most recent 30 or so years).

\section{Morphology}

Following Galen, mentioned above, an excellent example of progress comes from the work of Andreas Vesalius (1543, De Humani Corporis Fabrica). Here is the 
level of detail he achieved: "Professors of dissection usually divide the anterior brain, which they call the cerebrum, from the posterior brain, which they call the cerebellum: in turn, the anterior is normally divided into right and left. Not that the great masters of anatomy think that the brain is entirely divided..."

For me, the breakthrough to modern neurobiology occurred when chemical stains were discovered that would reveal microscopic details of nerve cells. The Italian scientist Camillo Golgi got a lucky break when nighttime cleaning personnel, servicing the hospital kitchen that Golgi had turned into a laboratory, knocked one of his human brain specimens into a slop bucket. Intrigued by the apparent staining of cells in that specimen, Golgi found that a key ingredient in turning some of the neurons dense-black was (and still is) silver nitrate. A brilliant exponent of Golgi stain-based nerve cell biology was the Spanish neuroanatomist Ramon y Cajal.

Cajal clearly stated the "neuron doctrine." The brain is not just a continuous string of fibers forming anastomoses to make never-ending nets. Instead, as Nobelist Cajal concluded, each nerve cell is an autonomous unit. "The neuron is the anatomical and physiological unit of the nervous system." And the rest is history. How do neurons talk to each other (Kruger and Otis 2007)? The Nobel winning physiologist Sir Charles Sherrington (1857-1952) "developed the concept of the synapse" and introduced modern neurophysiology in his 1932 book, "The Integrative Action of the Nervous System."

For decades the development and use of new neuroanatomical techniques dominated the scene. For example, a Dutch neuroanatomist, Walle J.H. Nauta, my teacher, who had survived World War II by eating tulip bulbs, came to the United States (MIT) and developed techniques for seeing very fine nerve fibers. This type of technical development led to our current state, when neuroscientists ambitiously are trying to map all the connections in the human brain.

\section{Physiology}

After microscopic techniques for looking at neurons gave our field a running start, scientists good at electrical recording invented what is called "neurophysiology." For example, in Britain, Lord Adrian received the Nobel Prize for showing, in 1938, how to record from individual nerve fibers. Later, tiny wire probes called microelectrodes were developed so that we could put them deep into the brain and record the electrical activity of individual neurons. Most prominent during the early years of this technical endeavor were David Hubel and Torsten Wiesel, who used such electrodes to elucidate the neurophysiology of the visual cortex. And, of course, recording in a non-invasive manner on the surface of the skin over the skull gives you "EEG:" electroencephalography of wave-like activity of the cerebral cortex so useful for clinical diagnosis, as in epilepsy or sleep problems. Sakmann's and Neher's Nobel prize-winning invention led to a modern development of the microelectrode: a tiny pipette that suctions onto the surface of an individual neuron, 
breaks through that membrane and records from inside the neuron. This is the "patch clamp" technique, which unveils the subtlest details of cross-membrane currents in nerve cells, especially in brain slices or in nerve cell culture.

\section{Chemistry}

Later still came the origins of neurochemistry. Of course, the discoveries of how neurotransmitters such as dopamine and acetylcholine are produced in neurons and how they are released at synapses and eventually broken down took center stage. The Nobel prize winner Julius Axelrod, running a large lab at the National Institutes of Health, became famous not only for his own work but also for mentoring an entire generation of neurochemical geniuses. One of those geniuses, Solomon Snyder, not only discovered opiate receptors in the brain but also could claim such a large number of advances in neurochemistry that the entire department of neuroscience at Johns Hopkins Medical School now is named after him.

Rita Levi-Montalcini's discovery of nerve growth factor (NGF) opened a new arena of neurochemistry in which peptide chemistry was paramount and led to the elucidation of families of related growth factors.

As DNA's chemistry and its regulation in gene expression became easier and easier to study, neuroscientists jumped on the bandwagon. For example, I was able to prove (reviewed in Pfaff 2002; Lee et al, 2009) that expression of a particular gene (that which codes for an estrogen receptor) in particular neurons of the brain (hypothalamic and preoptic neurons) is absolutely essential for specific instinctive behaviors (mating behavior and maternal behavior). And now the focus has shifted to the nuclear proteins that coat DNA in the neuron and regulate gene expression.

\section{Genetics, Genomics}

To manipulate gene expression in neuroendocrine cells, siRNA (small interfering RNA) was used to knock out a single gene (estrogen receptor-alpha) in specific neurons, which abolished all aspects of female reproductive behavior: in temporal order, lateral preoptic neurons (courtship behavior); ventromedial hypothalamic neurons (sex behavior; reviewed in Pfaff 2002); and medial preoptic neurons (maternal behavior; Ribeiro et al. 2012). These studies comprised a behaviorally relevant extension of nuclear hormone receptor chemistry in neuroendocrinology. 


\section{Cognitive Neuroscience}

As recently as 70 years ago, studies that dealt with complex behaviors-psychology, personality, and so forth-were dismissed by some as "soft." The scientific qualities of accuracy and precision were doubted for those fields. But the field of cognitive science has come a long way. As things began to improve, some 100 years ago, scientific approaches to the behaviors of animals were split into two parts. One approach, called ethology, most popular in Europe, usually treated the natural behaviors of animals in their natural environments. Ethology was rooted in biology. The other approach, experimental psychology, was more popular in America. Derived from physics, experimental psychological studies would feature wellcontrolled experiments in the laboratory to answer specific, precisely worded questions or to test formal hypotheses. Both of these approaches could be applied to human subjects. Finally, most famously, the Viennese neurologist Sigmund Freud originated the psychodynamic theory of the human mind and brain, psychoanalysis.

Cognitive neuroscientists often united these studies of behavior with the various neuroscientific methodologies and techniques mentioned above. Historically, brain lesions and their behavioral analyses came earliest. Well known currently, for example, is the patient HM. The Canadian neurosurgeon William Scoville removed most of his hippocampus on both sides of his brain to prevent continuing epileptic seizures. Then the Canadian psychologist Brenda Milner documented his permanent loss of memory for recent events. In other studies, human language was emphasized, as summarized by Chatterjee and Coslett (2014).

Looking back, the first great victory regarding language was the observation by the French neurologist Broca that loss of a delimited region on the lower side of the left frontal lobe impaired the production of speech. On the other hand, damage to a cortical area farther posterior, near the juncture of the temporal lobe and parietal lobe, again on the left side, would impair, in Heidi Roth's words "the acoustic images of words." Patients with this type of brain damage, studied by the German neurologist Carl Wernicke, could not identify or recognize normal speech. As you can imagine, these studies were based on small numbers of patients. More patients had to be studied, brain damage had to be better defined and the language analyses had to be more sophisticated. But Broca and Wernicke had paved the way.

From there neurologists and neuroscientists went on to initiate the study of all aspects of human behavior. My own lab has zeroed in on the most fundamental influence within the brain, a concept I call "generalized brain arousal," which is essential for initiation of all behaviors. On the other hand, neurologists tend to concentrate on specific disorders, such as epilepsy, autism, memory, and addiction. 


\section{Computational Approaches}

One branch of neuroscience came out of engineering, physics and mathematics. The theorem of McCulloch and Pitts, published in 1943, coupled with the interests of Alan Turing sparked the field alive. Then, in 1956, electrical engineers Claude Shannon (the inventor of information theory), John McCarthy and Marvin Minsky conceived and led the conference that generated the field of "artificial intelligence," the basis of sophisticated robotic behavioral regulation. All these scientists were applying techniques that had already been proven successful and using them for the potential understanding and mimicry of the brain's behavioral regulation.

Neuroscientific work now has reached such a level of precision that our data often can be treated with computations based on applied mathematics and statistics. Computational neuroscience can be divided into two parts: analysis and so-called "modeling," which means devising computer programs that are supposed to embody the essential features of some well-chosen groups of neurons in the brain. Both parts of computational neuroscience contribute to the type of artificial intelligence that regulates behaviors by robots and computations by neural networks.

As stated by Eve Marder, a prominent computational neuroscientist at Brandeis University, "computational models are invaluable and necessary in this task and yield insights that cannot otherwise be obtained. However, building and interpreting good computational models is a substantial challenge, especially so in the era of large datasets." Fitting detailed models to experimental data is difficult and often requires onerous assumptions, whereas more loosely constrained conceptual models that explore broad hypotheses and principles can yield more useful insights.

George Reeke, at Rockefeller University, envisions modeling of the brain as an obvious approach to answering questions all of us have about the brain: how are sensations, categorized, how are actions selected from a given repertoire, how is "motivation" to be conceived (2012)? It was the availability of computers that allowed academic researchers to construct ever more detailed and complicated models of the brain. Some neural modelers try actually to mimic neurons and neuronal systems faithfully, in detail, while others do not; instead, in Reeke's words, they just concentrate on devising "rule-based systems." In all cases, the equations neuronal modelers use to mimic neurons never match the full sophistication and flexibility of real neurons; neither are the circuitry properties of the human brain truly realized, even in the best models. These days, many neuroscientists are drawn into modeling, and thus a form of AI, because of the considerable number of free software modeling packages. The implication is that progress in neuronal modeling is accelerating. Nevertheless, as Reeke points out, the field is not without its shortcomings. For example, in some cases, the equations representing neurons and their connections are so abstract that they lose the properties of real neural systems. In other cases, neuronal modelers will run large numbers of trials 
and select some in which their favorite ideas work, which, of course, leads to false conclusions.

The operations of individual nerve cells and individual synapses comprise the irreducible base of neuronal modeling and have absorbed the attention of William Lytton, at State University of New York Medical Center. One starts with the nerve cell membrane. The equations that represent the membrane in the model contain the elements of electrical circuit theory: resistors and capacitors. Once those equations and the dynamic changes when electrical current flows, for example through sodium channels or calcium channels, are in place, you are ready to start building artificial "circuits." One example would be the modeling of a type of connection serving the passage of sensory information through the thalamus with its subsequent impact on the cerebral cortex, which can be modeled, as Lytton has done, using five types of "neurons" and nine types of connections between neurons.

\section{A Brief Survey of Emerging Techniques for Neuromodulation}

\section{Electrical}

One striking development demonstrated the use of the patient's own electrical waveform activity to move artificial limbs. While John Donoghue (Brown) was given a lot of credit for opening up this field, Miguel Nicolelis (Duke, Sao Paolo) has reported similar achievements. Dedicated to the use of helping injured war veterans with artificial limbs, Geoffrey Ling (DARPA) has shown effective control of artificial limbs in therapeutic settings.

On the sensory side of the CNS, some scientist/engineers are concerned with age-related macular degeneration. Retinal prostheses to help ameliorate this problem are a central concern of Sheila Nirenberg (Cornell), but the project has a dimension that goes well beyond prosthesis construction. Central to the solution is a deep understanding of the critical features of electrical signaling to the optic nerve. Working with the computational neuroscientist Jonathan Victor, Nierenberg is discovering the answer to that intellectual problem now.

\section{Chemical}

While the field of microfluidics has been applied extensively to sampling extremely small volumes of biological fluids, it will now become available for precise, timelimited local delivery of therapeutic substances in specific brain regions. For similar purposes, nanoparticles, lipid bilayered to cross blood-brain barrier, can be loaded 
up with chemicals intended for therapeutic purposes. Cationic liposomes, the positive charge offering the possibility of entry into cells, can be used likewise.

DREADDS - Designer Receptors Exclusively Activated by Designer Drugscan be genetically encoded so that they are expressed only in specific subpopulations of neurons, thus to bind pharmaceuticals..

\section{Genetic}

The applicability of optogenetics to the nervous system (Karl Deisseroth, Stanford) has been proven; it uses brief pulses of light to activate channel proteins that, in some cases, excite neurons and, in other cases, inhibit neurons. For example, inhibiting GABA neurons that, in turn, inhibit giant medullary reticular neurons can enhance recovery of consciousness from anesthesia, as measured by behavioral activation and by the activation of the cortical EEG.

\section{Viral}

Locally delivered by stereotaxically guided microinjection, adeno-associated viral particles (AAV) are outfitted with cell-selecting promoters to modify synthetic and electrical activities of selected subsets of neurons in that neuronal group (only).

\section{Computational}

In general, the use of temporal and spatial patterns of firing in the human brain's "connectome" requires big data computational efficacy. One specific example, viewing brain activity as a set of non-linear dynamic systems, would involve the identification and use of "attractor" states of neuronal circuitry. This project is being carried out in the context of the Obama BRAIN initiative.

\section{Special Opportunities for Manipulating the Unique Products of Neuroendocrine Neurons}

Because neuroendocrine neurons specifically produce small chemicals of surpassing importance for the governance of the physiology of the entire body, the possibility of using chemical, viral or genetic means to regulate their activity offers unique therapeutic opportunities. Seven examples: 
- GnRH: Gonadotropin releasing hormone controls all of reproductive physiology and reproductive behavior.

- GHRH: Growth hormone releasing hormone promotes the release of growth hormone from the pituitary.

- Somatostatin: Reduces the release of growth hormone from the pituitary.

- TRH: Thyrotropic releasing hormone facilitates the release of TSH from the pituitary Normal mentation and mood depend on thyroid hormone levels.

- CRH: Corticotropic releasing hormone facilitates the release of ACTH from the pituitary (stress response). In the brain, $\mathrm{CRH}$ (also known as $\mathrm{CRF}$ ) participates in circuits that govern stress-related behaviors.

- Oxytocin: In addition to regulating lactation and parturition, oxytocin participates in the initiation of maternal behavior and prosocial motivation.

- Vasopressin: Regulation of body water, blood pressure, blood volume. Vasopressin expression in certain forebrain neurons is known to facilitate aggression.

\section{This Volume}

As illustrated throughout this volume, stem cell biology is a fast-moving, young field with obvious therapeutic potential as well as technical and legal encumbrances. Following a didactic chapter intended for readers without a background in this area of medical science, several chapters will report striking advances. Between invitations for this project and the time of the meeting in Paris, one doctor moved from having a definite need for stem cell technology to publishing a pair of papers in the prestigious journals, Science and Cell. The meeting wrapped up with an overview from the Lovell-Badge group, the lab that 24 years ago reported the basis of sexual differentiation through the discovery of the SRY gene on the Y chromosome.

Open Access This chapter is distributed under the terms of the Creative Commons Attribution 4.0 International License (http://creativecommons.org/licenses/by/4.0/), which permits use, duplication, adaptation, distribution and reproduction in any medium or format, as long as you give appropriate credit to the original author(s) and the source, a link is provided to the Creative Commons license and any changes made are indicated.

The images or other third party material in this chapter are included in the work's Creative Commons license, unless indicated otherwise in the credit line; if such material is not included in the work's Creative Commons license and the respective action is not permitted by statutory regulation, users will need to obtain permission from the license holder to duplicate, adapt or reproduce the material.

\section{Further Reading}

Catani M, Sandrone S (2015) Brain renaissance: from Vesalius to modern neuroscience. Oxford University Press, Oxford 
Chatterjee A, Coslett HB (2014) The roots of cognitive neuroscience. Oxford University Press, Oxford

Dertouzos M et al (1974) Systems, networks and computation. McGraw-Hill, New York

Finger S (1994) Origins of neuroscience. Oxford University Press, Oxford

Gagnidze K, Weil ZM, Faustino LC, Schaafsma SM, Pfaff DW (2013) Early histone modifications in the ventromedial hypothalamus and preoptic area following oestradiol administration. J Neuroendocrinol 10:939-955

Hodges A, Turing A (1983) The enigma. Princeton University Press, Princeton

Kruger L, Otis TS (2007) Whither withered Golgi? A retrospective evaluation of reticularist and synaptic constructs. Brain Res Bull 72:201-207

Lee A et al (2009) In: Pfaff D (ed) Hormones, brain and behavior, 2nd edn. Academic Press, Elsevier, San Diego

Lytton W (2012) In: Pfaff D (ed) Chapters in neuroscience in the 21st century. Springer, Heidelberg

O'Leary T, Sutton AC, Marder E (2015) Computational models in the age of large datasets. Curr Opin Neurobiol 32C:87-94

Pfaff D (ed) (2002) Hormones, brain and behavior, 1st edn. Academic Press, Elsevier, San Diego

Pfaff D, Joels M (eds) (2016) Hormones, brain and behavior, 3rd edn. Elsevier, Cambridge

Pfaff D $(2012,2015)$ (ed) Neuroscience in the 21 st century (a five volume text free in poor countries, 1st edn. Heidelberg, Springer (Pfaff D, Volkow N (eds) 2nd edn. Heidelberg, Springer)

Reeke G (2012) In: Pfaff D (ed) Neuroscience in the 21st century. Springer, Heidelberg

Ribeiro AC, Musatov S, Shteyler A, Simanduyev S, Arrieta-Cruz I, Ogawa S, Pfaff DW (2012) siRNA silencing of estrogen receptor- $\alpha$ expression specifically in medial preoptic area neurons abolishes maternal care in female mice. Proc Nat Acad Sci USA 109(40):16324-16329

Wilson EG (2006) The melancholy android. SUNY Press, Albany 